\title{
Electronic and optical properties of single-walled carbon nanotubes under a uniform transverse electric field: A first-principles study
}

\author{
T. H. Cho, ${ }^{1,2}$ W. S. Su, ${ }^{1,3,4}$ T. C. Leung,,${ }^{1, *}$ Wei Ren, ${ }^{5}$ and C. T. Chan ${ }^{5}$ \\ ${ }^{1}$ Department of Physics, National Chung Cheng University, Chia-Yi 621, Taiwan \\ ${ }^{2}$ Department of Optometry, Shu Zen College of Medicine and Management, Kaohsiung 821, Taiwan \\ ${ }^{3}$ Department of Physics, National Cheng Kung University, Tainan 701, Taiwan \\ ${ }^{4}$ Center for General Education, Tainan University of Technology, Tainan 710, Taiwan \\ ${ }^{5}$ Department of Physics and William Mong Institute of Nano Science and Technology, Hong Kong University \\ of Science and Technology, Clear Water Bay, Hong Kong, China
}

(Received 10 March 2009; revised manuscript received 11 May 2009; published 17 June 2009)

\begin{abstract}
A systematic study on the effect of a transverse electric field on the optical properties of carbon nanotubes has been performed using density-functional calculations. The band gaps of the zigzag tubes decrease significantly as the electric field is increased. In the case of armchair tubes, the metallic band energy dispersion relation develops multivalleys at the Fermi level at high fields. The electric-field-induced changes in band dispersions lead to changes in optical properties as manifested in the dielectric functions. Some of the changes are not obvious. For example, a significant field-induced change in band gap can lead to small changes in dielectric functions in some special cases. Our results show that the armchair carbon nanotubes can be a promising material for electro-optical modulation device applications.
\end{abstract}

DOI: 10.1103/PhysRevB.79.235123 PACS number(s): 71.15.Mb, 78.67.Ch, 73.22.-f, 71.20.Tx

\section{INTRODUCTION}

Carbon nanotubes (CNTs) have received an increasing amount of interest owing to their novel properties and potential applications in nanodevices. ${ }^{1}$ It has been shown theoretically that the band gap of CNTs and boron nitride nanotubes (BNNTs) can be reduced by applying a transverse electric field. ${ }^{2}$ This band-gap modulation is due to the Stark effect, which is further confirmed experimentally by bias-dependent scanning tunneling microscopy and thin-film transistor configuration. ${ }^{3}$ On the other hand, an armchair-type CNT is metallic with two linear bands intersecting at the Fermi level regardless of its diameter. Using a $\pi$-band model, Li et al. ${ }^{4}$ have shown that the applied transverse electric field polarizes the nanotubes along the field direction, modifies band structure of armchair CNT, and results in the two lowest subbands with flattening near the Fermi level. As the field strength increases, the two lowest subbands show oscillatory bands with multiple nodes at the Fermi level. This interesting fieldinduced change in dispersion is verified in first-principles calculations, which are more accurate. Our recent firstprinciples studies suggested that the metallic band energy dispersion relation of armchair CNT indeed develops multivalleys at the Fermi level, which can lead to conduction amplification under a transverse electric field. ${ }^{5}$ The optical properties of CNTs have been investigated experimentally by optical absorption spectroscopy, ${ }^{6,7}$ by high-resolution electron-energy-loss spectroscopy in transmission, ${ }^{8}$ by reflectivity measurements, ${ }^{9}$ and also by spectrofluorimetric measurements. ${ }^{10}$ Calculations of the optical properties of CNTs have been reported intensively by $\pi$-band model, ${ }^{11}$ modified tight-binding (TB) model, ${ }^{12}$ all-valence TB models, ${ }^{13,14}$ and ab initio models ${ }^{15}$ showing that the absorption spectra exhibit rich characteristic peaks due to the onedimensional Van Hove singularities. Although the gap of BNNTs is reduced significantly by the extended field, the change in the optical absorption spectrum was found to be less pronounced. ${ }^{16}$ However, to our knowledge, the effect of the transverse electric field on the optical properties of CNTs has not been studied yet, and this will be the focus of this paper.

\section{METHOD OF CALCULATION}

The calculations are performed within the framework of local-density approximation, ${ }^{17}$ using highly accurate fullpotential projected augmented wave (PAW) method, ${ }^{18}$ as implemented in the VASP package. ${ }^{19}$ A supercell geometry was adopted so that the nanotubes are arranged in a square array with intertube distance equal to $10 \AA$. At such arrangement, the tube-tube interactions are very small so that the tubes can be treated as independent entities. The length of the unit cell along the tube axis is determined by minimizing the total energy of the system. A large plane-wave cutoff of $358.2 \mathrm{eV}$ was used throughout. The $k$-point sampling was set at $1 \times 1 \times 200$. All atoms were fully relaxed without the external field until the maximum magnitude of the force acting on the atoms was smaller than $0.02 \mathrm{eV} / \AA$. The changes in the geometry induced by the external field are neglected, as they are always very small and have negligible effects on the optical properties. The Fermi energy is determined using standard Gaussian smearing method with the width of the smearing set at $0.1 \mathrm{eV}$. In the calculation, the tube axis is along the $x$ direction and the external electric field is along the $z$ direction. The external electric field is imposed by putting a positive charge sheet in the vacuum on one side and a negative charge sheet on another side of the tube.

In the random-phase approximation and neglecting the local-field corrections, the imaginary part of the dielectric function with radiation polarized in the $\alpha$ direction is given as a function of the photon energy $\hbar \omega$ expressed as follows: ${ }^{20}$ 

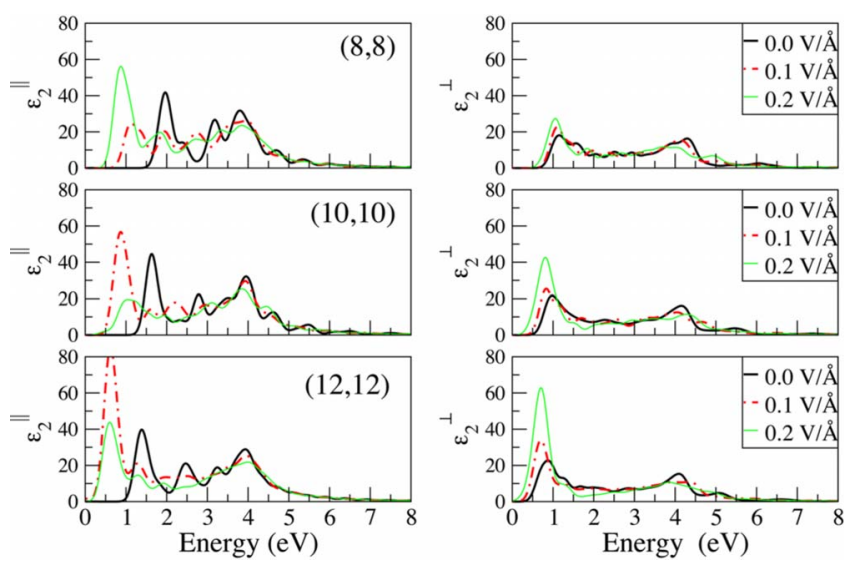

FIG. 1. (Color online) The imaginary part of the dielectric function of armchair nanotubes for light polarized parallel (left panel) and perpendicular (right panel) to the nanotubes axis under external electric fields $\vec{E}$ of $0.0,0.1$, and $0.2 \mathrm{~V} / \AA$.

$$
\varepsilon_{2}(\omega)=\frac{4 \pi^{2}}{\Omega \omega^{2}} \sum_{i \in \mathrm{VB}, j \in \mathrm{CB}} \sum_{k} \omega_{k} p_{i j}^{\alpha}(k) \delta\left(\varepsilon_{k j}-\varepsilon_{k i}-\omega\right),
$$

where $\Omega$ is the effective unit cell volume of the nanotubes as proposed by Machon et al. ${ }^{21}$ and VB and CB denote the conduction and valence bands, respectively. The matrix elements $p_{i j}^{\alpha}(\vec{k})=\left|\left\langle i, \vec{k}\left|\hat{p}_{\alpha}\right| j, \vec{k}\right\rangle\right|^{2}$ are obtained from the selfconsistent band structures within the PAW formalism ${ }^{22}$ and $\omega_{k}$ is the $k$ th point weighting. Here, $\hat{p}_{\alpha}$ is the $\alpha$ component of the momentum operator. The initial and final states $|i, \vec{k}\rangle$ and $|j, \vec{k}\rangle$ indicate valence and conduction bands, respectively. The real part of the dielectric function $\varepsilon_{1}(\omega)$ is obtained by the Kramers-Kronig transformation.

\section{RESULTS}

We first considered the effect of the applied transverse electric field on the optical properties of armchair nanotubes. The imaginary part of the dielectric function $\varepsilon_{2}^{\|}$(and $\varepsilon_{2}^{\perp}$ ), which correspond to light polarized parallel (and perpendicular) to the nanotubes axis, are evaluated explicitly in this study. The left panel of Fig. 1 shows the spectrum of $(8,8)$, $(10,10)$, and $(12,12)$ nanotubes under different strengths of transverse electric fields. For the case at the zero-applied electric field, the spectrum consists of a few distinct peaks that are related to dipole-allowed transitions between states near the Van Hove singularities that are found in the quasione-dimensional density of states (DOS). The energy positions of the peaks related to transitions between the first and second pairs of DOS singularities (designated as $E_{11}$ and $E_{22}$ ) are observed at 1.96 and $3.18 \mathrm{eV}$ for $(8,8), 1.64$ and $2.78 \mathrm{eV}$ for $(10,10)$, and 1.40 and $2.46 \mathrm{eV}$ for $(12,12)$ nanotubes. The results are in good agreement with recent DFT calculations. ${ }^{15}$ Under the applied electric field, the spectra displayed apparent redshifts and broadening. The intensity of the original $E_{11}$ peak is strongly suppressed by the applied electric field. In addition, a strong peak emerges at about half the value of $E_{11}$, and a small peak emerges at about half the value of $\left(E_{11}+E_{22}\right)$. The high sensitivity of the optical spectrum to an
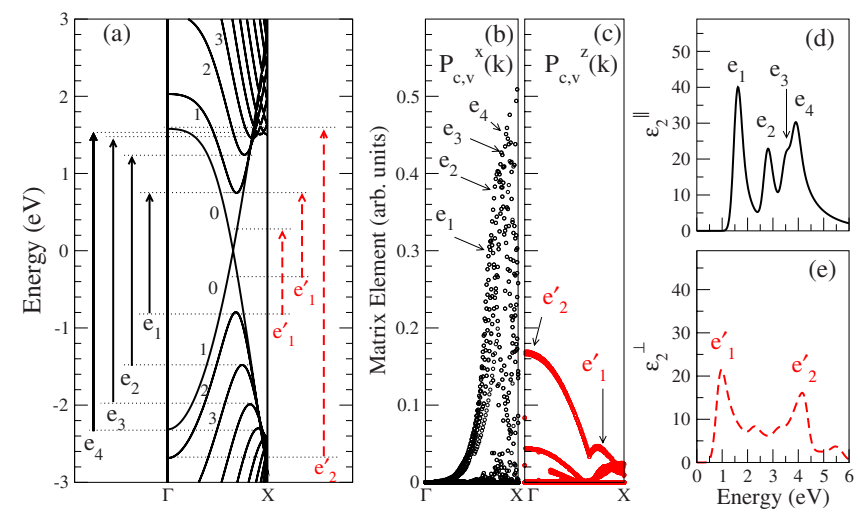

FIG. 2. (Color online) The band structures, matrix elements, and the $\varepsilon_{2}$ spectrum for $(10,10)$ carbon nanotube under zero external field.

applied electric field makes the armchair carbon nanotubes a plausible material for optoelectronic application.

The right panel of Fig. 1 shows the $\varepsilon_{2}^{\perp}$ spectra of $(8,8)$, $(10,10)$, and $(12,12)$ nanotubes under different strengths of transverse electric fields. The spectra look like flat platforms with humps on both edges. The bandwidth of the spectra extends wider as the diameter of the nanotubes becomes larger. This is also in good agreement with the previous calculations. ${ }^{15}$ As the external field strength increases, the intensity of the first peak is enhanced significantly, while the energy position of this peak is shifted toward lower energies.

To better understand the field effect on the optical transitions in the armchair tube, we investigated the modification of the band structures and the matrix elements under the influence of externally imposed electric field. The contribution to the dielectric function is determined by two factors, namely, the joint density of states and the matrix elements for the corresponding valence and conduction states. When a Van Hove singularity appears in the joint density of states and the matrix elements between the corresponding valence and conduction states have a nonzero value, a corresponding peak will show up in the dielectric function spectrum. Figure 2 shows the band structures, the matrix elements, and the $\varepsilon_{2}$ spectrum for $(10,10)$ carbon nanotubes without an external electric field. The corresponding optical transitions are indicated schematically in Fig. 2. We will first look at the case of light polarized parallel to the tube axis. We see that the $\varepsilon_{2}^{\|}$ spectrum consists of four peaks at 1.64, 2.78, 3.60, and 3.96 $\mathrm{eV}$, which are in good agreement with previous results in the literature. ${ }^{23}$ Figure 2(b) shows the matrix element $p_{i j}^{\alpha}(k)$ for the transition between the ten highest occupied bands (band indices 71-80 in the calculation) and the ten lowest unoccupied bands (band indices 81-90). We note that that the matrix elements from $E_{\mu^{\prime}}^{\nu} \rightarrow E_{\mu}^{c}$ (designated as $E_{\mu^{\prime} \mu}$ ) have a nonvanishing value only for the energy subbands $\mu=\mu^{\prime}$, where the subband indices of $E_{\mu^{\prime}}^{\nu}$ and $E_{\mu}^{c}$ are taken symmetrically around the Fermi energy is indicated in Fig. 2(a). As a function of $k$, the matrix elements vanish at $\Gamma$ point, and the value grows and reaches a maximal value at the Van Hove singular point as shown in Fig. 2(b). The maximal value of the matrix element for $E_{\mu^{\prime} \mu}$ transition increases as the value of subband index $\mu$ increases. 

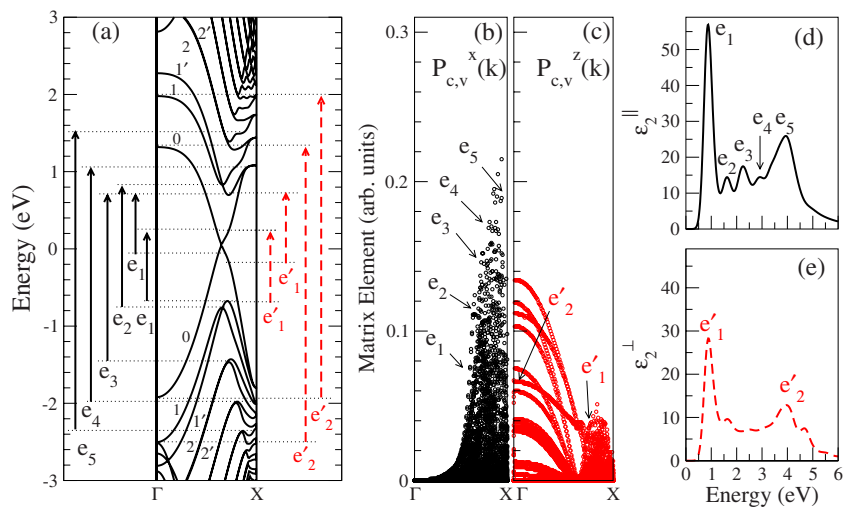

FIG. 3. (Color online) The band structures, matrix elements, and the $\varepsilon_{2}$ spectrum for $(10,10)$ carbon nanotube under an external field $\vec{E}$ of $0.1 \mathrm{~V} / \AA$.

For the case of light polarized perpendicular to the tube axis, the $\varepsilon_{2}^{\perp}$ spectrum is made up of a double humplike shape with two main peaks located at 0.98 and $4.16 \mathrm{eV}$. The matrix elements are nonzero only for the $E_{\mu, \mu \pm 1}$ transition. The $e_{1}^{\prime}$ peaks are contributed by the $E_{01}$ and $E_{10}$ transitions at $\Gamma$ point, and the $e_{2}^{\prime}$ peak is contributed by the $E_{01}$ and $E_{10}$ transitions at the valence-band maximum (or conductionband minimum) $k=0.66 \frac{\pi}{a}$. In our calculations, the static external applied field is screened as the calculations are done fully self-consistently in the presence of the external field. However, in the presence of light, the dynamic screening effects are not included in the calculations because the local field corrections are not incorporated, and local field effects can reduce the dielectric response when the electric field is perpendicular to the tube axis. ${ }^{24}$

Next, we study the effect of the external electric field. Figure 3 shows the band structures, the matrix elements, and the $\varepsilon_{2}$ spectrum for $(10,10)$ carbon nanotubes under an external field of $0.1 \mathrm{~V} / \AA$. The external field induces splitting of the double degenerate electronic bands away from the Fermi energy. Because of the electronic band mixing caused by the external field, the $E_{\mu, \mu \pm 1}$ and $E_{\mu, \mu \pm 2}$ transitions are now allowed.

For the $\varepsilon_{2}^{\|}$spectrum, the values of the matrix elements are reduced by the external field. The $e_{1}, e_{2}, e_{3}, e_{4}$, and $e_{5}$ peaks
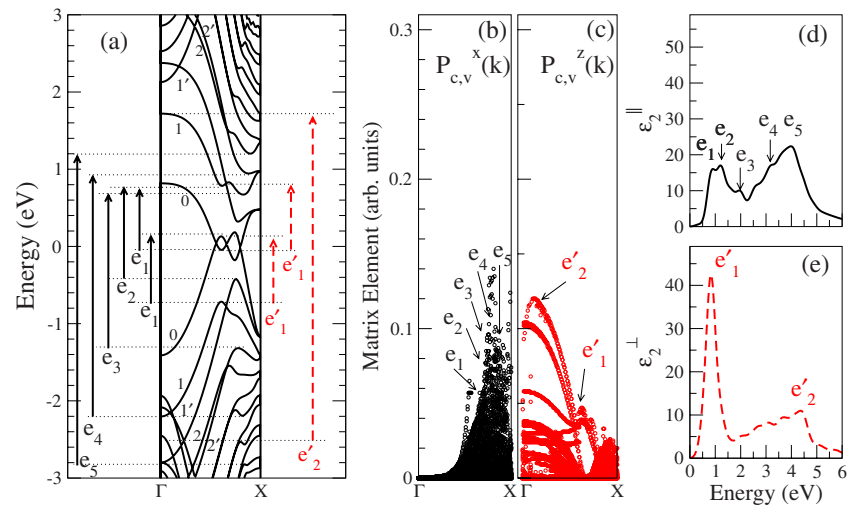

FIG. 4. (Color online) The band structures, the matrix elements, and the $\varepsilon_{2}$ spectrum for $(10,10)$ carbon nanotube under an external field $\vec{E}$ of $0.2 \mathrm{~V} / \AA$.
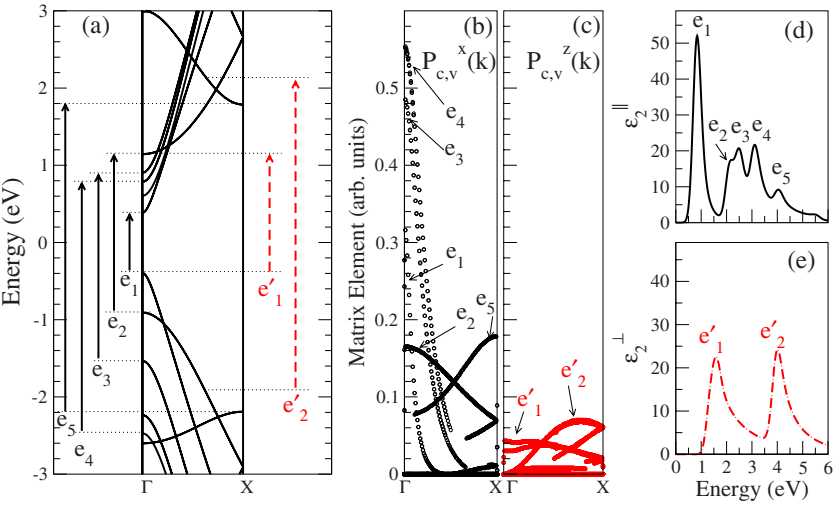

FIG. 5. (Color online) The band structures, the matrix elements, and the $\varepsilon_{2}$ spectrum for $(10,0)$ carbon nanotube under zero external field.

are contributed by the $\left(E_{01^{\prime}}, E_{1^{\prime} 0}\right),\left(E_{1^{\prime} 1}\right),\left(E_{2^{\prime} 1}\right),\left(E_{3^{\prime} 1}\right)$ and $\left(E_{24^{\prime}}, E_{2^{\prime} 4}, E_{33^{\prime}}, E_{3^{\prime} 3}, E_{42^{\prime}}, E_{4^{\prime} 2}\right)$ transitions near the valenceband maximum or conduction-band minimum, respectively. On the other hand, the values of the matrix elements for the $\varepsilon_{2}^{\perp}$ spectrum are only slightly reduced by the external field. The $e_{1}^{\prime}$ and $e_{2}^{\prime}$ peaks are contributed by the $\left(E_{1^{\prime} 0}, E_{01^{\prime}}\right)$ transitions near the valence-band maximum or conduction-band minimum and the $\left(E_{01}, E_{10}\right)$ transitions near the $\Gamma$ point.

When the external field is increased to $0.2 \mathrm{~V} / \AA$, the singly degenerate bands crossing the Fermi level display oscillatory bends, intersecting the $E_{F}=0$ line at three points as shown in Fig. 4. The matrix elements for the $\varepsilon_{2}^{\|}$and $\varepsilon_{2}^{\perp}$ spectra are further reduced by the external field. The fine structures in the spectra are washed out owing to the splitting of the bands that are degenerate at zero external field.

In the following, we consider the effect of the external field on the electronic and optical properties of zigzag carbon nanotubes. The band structures, the matrix elements, and the $\varepsilon_{2}^{\|}$spectrum for the $(10,0)$ carbon nanotube under external fields $\vec{E}$ of $0.0,0.1$, and $0.2 \mathrm{~V} / \AA$ are shown in Figs. 5-7, respectively. We first consider the case of light polarized parallel to the tube axis. Without an external field, the $\varepsilon_{2}^{\|}$spectrum consists of a strong peak at $0.84 \mathrm{eV}$ and four weaker peaks at 2.20, 2.48, 3.10, and $4.04 \mathrm{eV}$. The $e_{1}, e_{2}, e_{3}$, and $e_{4}$
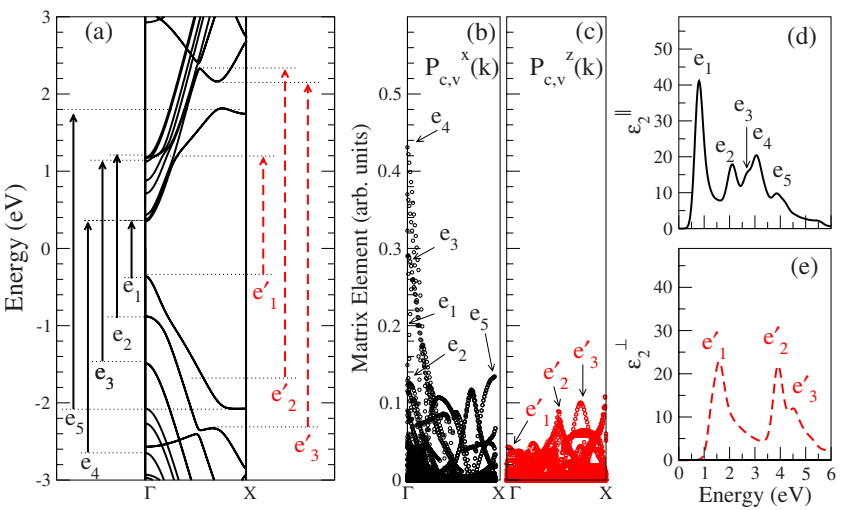

FIG. 6. (Color online) The band structures, the matrix elements, and the $\varepsilon_{2}$ spectrum for $(10,0)$ carbon nanotube under an external field $\vec{E}$ of $0.1 \mathrm{~V} / \AA$. 

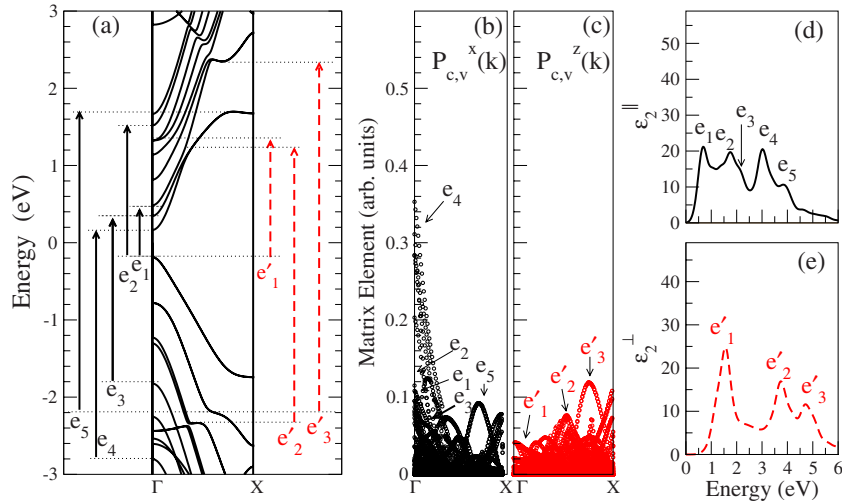

FIG. 7. (Color online) The band structures, the matrix elements, and the $\varepsilon_{2}$ spectrum for $(10,0)$ carbon nanotube under an external field $\vec{E}$ of $0.2 \mathrm{~V} / \AA$.

peaks are contributed by the $E_{11}, E_{25}, E_{34}$, and $\left(E_{52}, E_{34}\right)$ transitions near the zone center. It is interesting to note that the matrix elements of the above transitions have a maximum value at $\Gamma$ points. On the other hand, the $e_{5}$ peak is contributed by the $E_{66}$ transitions near the $X$ point where the matrix element has a maximum value. As the field strength increases, the band gap is reduced significantly. At the same time, the onset of the first peak of the $\varepsilon_{2}^{\|}$spectrum is shifted toward lower energies. A broken symmetry of the $(10,0)$ tube caused by the external field induces a gap opening at the crossing of bands, such as $E_{6}^{\nu}$ and $E_{2}^{\nu}$ (or $E_{5}^{c}$ and $E_{6}^{c}$ ) at $k$ $=0.76 \frac{\pi}{a}$. The external field also induces the splitting of several bands that are degenerate at zero electric field. Because of the electronic band mixing caused by the external field, transitions that are forbidden at zero external field become allowed, which can be seen by comparing the (b) and (c) panels in Figs. 7 and 8 with those of Fig. 6. With increasing external fields, the fine structure of the $\varepsilon_{2}^{\|}$spectrum is washed out because of the opening of the crossing bands, the splitting of the degenerated states, and the mixing of the character of the bands.

With increasing external field, the $e_{1}$ and $e_{5}$ peaks shift to slightly lower energy, the $e_{2}$ and $e_{4}$ peaks remain almost constant, and the $e_{3}$ peak is washed out. For $\vec{E}=0.2 \mathrm{~V} / \AA$, two extra peaks appear at 1.74 and $3.92 \mathrm{eV}$, which are caused by the modification of the band structure. In the case of light polarized perpendicular to the tube axis, there are two peaks located at 1.58 and $4.02 \mathrm{eV}$, respectively, at the zero external field. The $e_{1}^{\prime}$ peak is contributed by the $E_{15}$ transition at the $\Gamma$ point and the $E_{21}$ transition at about $0.1 \Gamma X$ away from the $\Gamma$ point. The $e_{2}^{\prime}$ peak is contributed by the $E_{26}$ transition in the region where the $e_{2}^{\nu}$ and $e_{6}^{c}$ bands are relatively parallel. As the field strength increases, the $e_{1}^{\prime}$ peak remains constant and the $e_{2}^{\prime}$ peak shifts to lower energy. An extra peak appears at $4.5 \mathrm{eV}$ for $\vec{E}=0.1 \mathrm{~V} / \AA$. The peak shifts to $4.72 \mathrm{eV}$ when the field is increased to $0.2 \mathrm{~V} / \AA$.

Figure 8 shows the $\varepsilon_{2}^{\|}$and $\varepsilon_{2}^{\perp}$ spectra of $(8,0),(10,0)$, and $(12,0)$ nanotubes under different strengths of external field. By increasing the external field, the transition peaks shift to lower energy and the intensity of the peak is suppressed. However, we note that the change, in particular the downshift of the lowest energy absorption peak, is less dramatic
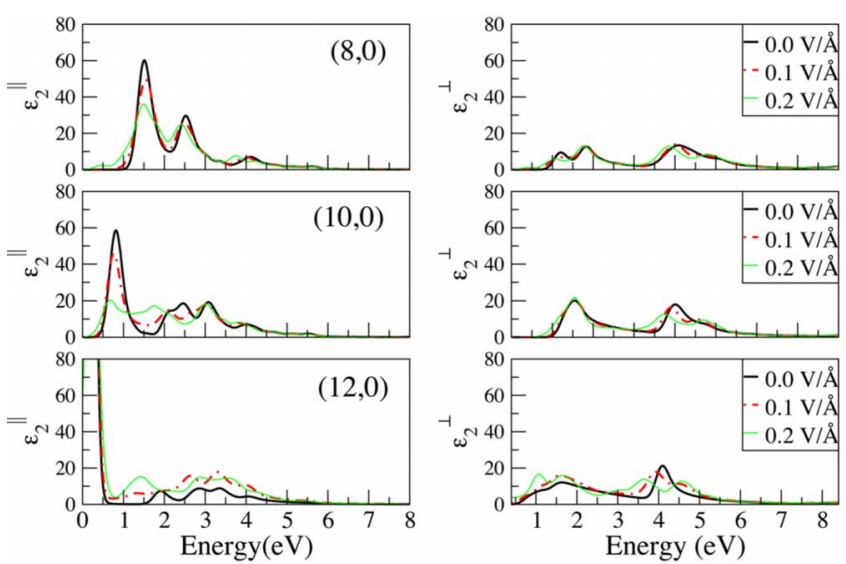

FIG. 8. (Color online) The imaginary part of the dielectric function of zigzag nanotubes for light polarized parallel (left panel) and perpendicular (right panel) to the nanotubes axis under external electric field $\vec{E}$ of $0.0,0.1$, and $0.2 \mathrm{~V} / \AA$.

than in the case of armchair tubes, which is evident when we compare Fig. 8 with Fig. 1. The fine structure of the spectrum is washed out owing to the splitting of the bands that are degenerate at zero electric field.

\section{CONCLUSIONS}

In conclusion, we have systematically investigated the effect of a transverse electric field on the band structure and optical properties of the carbon nanotubes by densityfunctional calculation. The matrix elements over the whole one-dimensional Brillouin zone of armchair and zigzag carbon nanotubes under different electric field strengths are presented and discussed. The band gap of zigzag tube decreases significantly as the electric field increases. We thus observe a rather sharp contrast in the field-induced behavior between zigzag and armchair tubes. In the case of zigzag tubes, the band structures are conspicuously changed by the field. In particular, the gap is reduced significantly; however, the effect of the electric field on the dielectric function is less pronounced. On the other hand, the armchair tube remains metallic under external electric field, but the dielectric functions are modified significantly owing to the subband mixing caused by the external field. The high sensitivity of the optical spectrum to an applied electric field makes the armchair carbon nanotubes a plausible material for optoelectronic applications.

\section{ACKNOWLEDGMENTS}

We acknowledge the National Center for Theoretical Sciences (NCTS) in Taiwan and financial support from the National Science Council of Taiwan (NSCT) under Grant Nos. NSC-96-2112-M194-012-MY3, NSC-97-2120-M002007, and NSC-97-2811-M-165-002, from the Research Grants Council of Hong Kong through Grant No. HKUST9/ CRF/08, and HKUST through Grant No. RPC06/07.SC21. We acknowledge a grant of computer time at the National Center for High Performance Computing in Taiwan. 
*Corresponding author; tcleung@phy.ccu.edu.tw

${ }^{1}$ S. Iijima, Nature (London) 354, 56 (1991).

${ }^{2}$ C.-W. Chen, M.-H. Lee, and Y.-T. Lin, Appl. Phys. Lett. 89, 223105 (2006).

${ }^{3}$ M. Ishigami, J. D. Sau, S. Aloni, M. L. Cohen, and A. Zettl, Phys. Rev. Lett. 94, 056804 (2005).

${ }^{4}$ Yan Li, Slava V. Rotkin, and Umberto Ravaioli, Nano Lett. 3, 183 (2003).

${ }^{5}$ Wei Ren, T. H. Cho, T. C. Leung, and C. T. Chan, Appl. Phys. Lett. 93, 142102 (2008).

${ }^{6}$ M. Ichida, S. Mizuno, Y. Tani, Y. Satio, and A. Nakamura, J. Phys. Soc. Jpn. 68, 3131 (1999).

${ }^{7}$ Z. M. Li, Z. K. Tang, H. J. Liu, N. Wang, C. T. Chan, R. Saito, S. Okada, G. D. Li, J. S. Chen, N. Nagasawa, and S. Tsuda, Phys. Rev. Lett. 87, 127401 (2001).

${ }^{8}$ R. Kuzuo, M. Terauchi, and M. Tanaka, Jpn. J. Appl. Phys., Part 2 31, L1484 (1992); V. P. Dravid, X. Lin, Y. Wang, A. Lee, J. B. Ketterson, and R. P. H. Chang, Science 259, 1601 (1993); P. M. Ajayan, S. Iijima, and T. Ichihashi, Phys. Rev. B 47, 6859 (1993); L. A. Bursill, P. A. Stadelmann, J. L. Peng, and S. Prawer, ibid. 49, 2882 (1994).

${ }^{9}$ F. Bommeli, L. Degiorgi, P. Wachter, W. S. Bacsa, W. A. de Heer, and L. Forro, Solid State Commun. 99, 513 (1996).

${ }^{10}$ S. M. Bachilo, M. S. Strano, C. Kittrell, R. H. Hauge, R. E. Smalley, and R. B. Wiesman, Science 298, 2361 (2002).

${ }^{11}$ X. Blase, L. X. Benedict, E. L. Shirley, and S. G. Louie, Phys.
Rev. Lett. 72, 1878 (1994).

${ }^{12}$ A. Hagen and T. Hertel, Nano Lett. 3, 383 (2003).

${ }^{13}$ J. X. Cao, X. H. Yan, J. W. Ding, and D. L. Wang, J. Phys.: Condens. Matter 13, L271 (2001).

${ }^{14}$ I. Milošević, T. Vuković, S. Dmitrović, and M. Damnjanović, Phys. Rev. B 67, 165418 (2003).

${ }^{15}$ G. Y. Guo, K. C. Chu, D. S. Wang, and C. G. Duan, Phys. Rev. B 69, 205416 (2004).

${ }^{16}$ C. Attaccalite, L. Wirtz, A. Marini, and A. Rubio, Phys. Status Solidi B 244, 4288 (2007).

${ }^{17}$ W. Kohn and L. J. Sham, Phys. Rev. 140, A1133 (1965).

${ }^{18}$ P. E. Blöchl, Phys. Rev. B 50, 17953 (1994); G. Kresse and D. Joubert, ibid. 59, 1758 (1999).

${ }^{19}$ G. Kresse and J. Hafner, Phys. Rev. B 47, 558 (1993); 49, 14251 (1994); G. Kresse and J. Furthmüller, Comput. Mater. Sci. 6, 15 (1996).

${ }^{20}$ H. Ehrenreich and M. H. Cohen, Phys. Rev. 115, 786 (1959).

${ }^{21}$ M. Machón, S. Reich, C. Thomsen, D. Sánchez-Portal, and P. Ordejón, Phys. Rev. B 66, 155410 (2002).

${ }^{22}$ B. Adolph, J. Furthmüller, and F. Bechstedt, Phys. Rev. B 63, 125108 (2001).

${ }^{23}$ X. Yang, G. Wu, J. Zhou, and J. Dong, Phys. Rev. B 73, 235403 (2006).

${ }^{24}$ L. X. Benedict, S. G. Louie, and M. L. Cohen, Phys. Rev. B 52, 8541 (1995). 